\title{
Cross-correlation based methods for estimating the functional connectivity in cortical networks
}

\author{
Alessandro Ide ${ }^{* 1,4}$, Michela Chiappalone ${ }^{1,2}$, Luca Berdondini³, \\ Vittorio Sanguineti ${ }^{4}$ and Sergio Martinoia ${ }^{1}$
}

\begin{abstract}
Address: ${ }^{1}$ Neuroengineering and Bio-nano Technology group - NBT, DIBE, University of Genova, Genova, Italy, ${ }^{2}$ Italian Institute of Technology - IIT, Unit of Neuroscience and Brain Technology Genova, Italy, ${ }^{3}$ Sensors, Actuators and Microsystems Laboratory, IMT, University of Neuchâtel, Neuchâtel, Switzerland and ${ }^{4}$ Neurolab, DIST, University of Genova, Genova, Italy

Email: Alessandro Ide* - noriaki@dist.unige.it

* Corresponding author
\end{abstract}

from Sixteenth Annual Computational Neuroscience Meeting: CNS*2007

Toronto, Canada. 7-12 July 2007

Published: 6 July 2007

BMC Neuroscience 2007, 8(Suppl 2):P63 doi:I0.I I86/I47I-2202-8-S2-P63

(C) 2007 Ide et al; licensee BioMed Central Ltd.

\section{Introduction}

Identification of the causal relationships between pairs of neurons plays an important role in the study of synaptic interactions within the nervous system at the population level. The simplest approach uses the cross-correlation function between pairs of spike trains. However, crosscorrelograms cannot tell whether the observed peaks or troughs in the correlation function derive from either direct or indirect connections, or result from a common input. This limitation can be partly overcome with the notion of partial correlation or conditional firing probability $[1,2]$.

\section{Methods}

Dissociated cortical neurons were obtained from rat embryos and plated into high-density microelectrode arrays (MEAs). Functional connectivity is estimated using cross-correlation based techniques and partial-correlation. To determine a connection, and also its strength, between two electrodes we calculated the area under the highest peak near to zero. Its latency determines the direction in the transmission of information.

\section{Results}

Cross-correlation based methods measure the direction of a possible connection between a pair of electrodes meanwhile, partial correlation comes out not only with direction but also eliminates indirect connections and gives the real strength between two channels. However, partialization presents some limitations when the number of neurons and connectivity of the network increases. Depending on how big is the network, partial correlation can show unreliable results or even breakdown in the identification of synaptic connections. In this work, we analyzed data obtained from cortical cultures coupled to high-density MEAs and we compared cross-correlation based techniques with partial-correlation analysis.

\section{Discussion}

Cross-correlation based methods are useful tools to estimate functional connectivity at the population level. Standard cross-correlation is applied just between pairs of electrodes and does not consider the entire network. It is the simplest method to infer about functional connectivity and obtain a general overview of the network map. On the other hand, partial correlation gives more details about the connectivity. Both methods can be used to study the development of the network or changes in the network behavior after electrical and/or chemical stimulation.

\section{References}

I. Eichler M, Dahlhaus R, Sandkuhler J: Partial correlation analysis for the identification of synaptic connections. Biol Cybern 2003, 89(4):289-302.

2. Feber J, Rutten WLC, Stegenga J, Wolters PS, Ramakers GJ, van Pelt $\mathrm{J}$ : Cultured cortical networks described by conditional firing 
probabilities. 5th international meeting on substrate-integrated micro electrode arrays, 4-7 Jul 2006, Reutlingen, Germany : pp. 67-70.

Publish with Bio Med Central and every scientist can read your work free of charge

"BioMed Central will be the most significant development for disseminating the results of biomedical research in our lifetime. " Sir Paul Nurse, Cancer Research UK

Your research papers will be:

- available free of charge to the entire biomedical community

- peer reviewed and published immediately upon acceptance

- cited in PubMed and archived on PubMed Central

- yours - you keep the copyright 